\title{
Spectroscopic Study of the Early-Type Binary HX Vel A
}

\author{
Burcu Özkardeş ${ }^{1,2}$, Derya Sürgit ${ }^{1,2}$, Ahmet Erdem ${ }^{1,2}$, \\ Edwin Budding ${ }^{3}$, Faruk Soydugan ${ }^{1,2}$, and Osman Demircan ${ }^{1,2}$ \\ ${ }^{1}$ Astrophysics Research Center and Observatory, Çanakkale Onsekiz Mart University, \\ Terzioğlu Kampüsü, TR-17020, Çanakkale, Turkey \\ ${ }^{2}$ Department of Physics, Faculty of Arts and Sciences, Çanakkale Onsekiz Mart University, \\ Terzioğlu Kampüsü, TR-17020, Çanakkale, Turkey \\ email: burcu@comu.edu.tr, dsurgit@comu.edu.tr, aerdem@comu.edu.tr, \\ f soydugan@comu.edu.tr, demircan@comu.edu.tr \\ ${ }^{3}$ Carter National Observatory, PO Box 2909, Wellington, New Zealand \\ email: budding@xtra.co.nz
}

\begin{abstract}
This paper presents high resolution spectroscopy of the HX Vel (IDS 08390-4744 AB) multiple system. New spectroscopic observations of the system were made at Mt. John University Observatory in 2007 and 2008. Radial velocities of both components of HX Vel A were measured using gaussian fitting. The spectroscopic mass ratio of the close binary was determined as $0.599 \pm 0.052$, according to a Keplerian orbital solution. The resulting orbital elements are $a_{1} \sin i=0.0098 \pm 0.0003 \mathrm{AU}, a_{2} \sin i=0.0164 \pm 0.0003 \mathrm{AU}, M_{1} \sin ^{3} i=1.19 \pm 0.07 \mathrm{M} \odot$ and $M_{2} \sin ^{3} i=0.71 \pm 0.04 \mathrm{M}_{\odot}$.
\end{abstract}

Keywords. methods: data analysis, techniques: spectroscopic, stars: early-type, (stars:) binaries (including multiple): close, stars: individual (HX Vel A)

\section{Spectroscopic observations and reductions}

High-resolution spectra of HX Vel were taken at the Mt John University Observatory (MJUO, New Zealand) in September and October 2007 and in December 2008, using the HERCULES (High Efficiency and Resolution Canterbury University Large Echelle Spectrograph) and a $4 \mathrm{k} \times 4 \mathrm{k}$ Spectral Instruments 600 series (SI600s) CCD camera attached to the 1-m McLellan telescope. Our observations were made choosing a slitless $100 \mu \mathrm{m}$ fiber cable with the second option of $\mathrm{R}=40000$, considered to be better adjusted to the mean seeing value (3.5 arcsec) at MJUO as given by Hearnshaw et al. (2002). A total of 20 spectra have been collected during six observing nights in 2008, while 7 high-resolution spectra of the binary were obtained during four observing nights between September and October 2007.

For all observations, a thorium-argon lamp spectrum for wavelength calibration was taken before and after each stellar exposure. White lamp spectra for flat-fielding were also taken every night. All spectra obtained were reduced using the HRSP (Hercules Reduction Software Package, HRSP: Skuljan \& Wright 2007) software.

\section{Radial velocities and orbital solution}

Radial velocities (RVs) of both components of HX Vel A binary were measured using gaussian fittings to the selected spectral lines, with the aid of the 'splot' task of IRAF. According to the B1.5V spectral type of the binary (SIMBAD), there should be strong neutral He and hydrogen lines of the Balmer series in its observed spectra. We found four 
Table 1. Spectral orders and stellar lines used in RVs measurements of HX Vel A.

\begin{tabular}{ccc}
\hline Order No & Wavelength Interval $(\AA)$ & Dominant Spectral Lines \\
\hline 85 & $6647-6715$ & He I $(6678.151 \AA)$ \\
97 & $5840-5907$ & He I $(5875.989 \AA)$ \\
116 & $4884-4939$ & He I $(4921.931 \AA)$ \\
127 & $4453-4508$ & He I $(4471.480 \AA)$ \\
\hline
\end{tabular}

Table 2. Spectroscopic orbital parameters of HX Vel A.

\begin{tabular}{lllr}
\hline Parameter & Value & Parameter & Value \\
\hline$P($ days $)$ & $1.12447($ fixed $)$ & $V_{\gamma}(\mathrm{km} / \mathrm{s})$ & $26 \pm 3$ \\
$T_{0}(\mathrm{HJD}+2448501)$ & $0.0833 \pm 0.0046$ & $M_{1} \sin ^{3} i\left(\mathrm{M}_{\odot}\right)$ & $1.19 \pm 0.07$ \\
$K_{1}(\mathrm{~km} / \mathrm{s})$ & $95 \pm 3$ & $M_{2} \sin ^{3} i\left(\mathrm{M}_{\odot}\right)$ & $0.71 \pm 0.04$ \\
$K_{2}(\mathrm{~km} / \mathrm{s})$ & $158 \pm 3$ & $a_{1} \sin i(\mathrm{AU})$ & $0.0098 \pm 0.0003$ \\
$q$ & $0.599 \pm 0.052$ & $a_{2} \sin i(\mathrm{AU})$ & $0.0164 \pm 0.0003$ \\
\hline
\end{tabular}
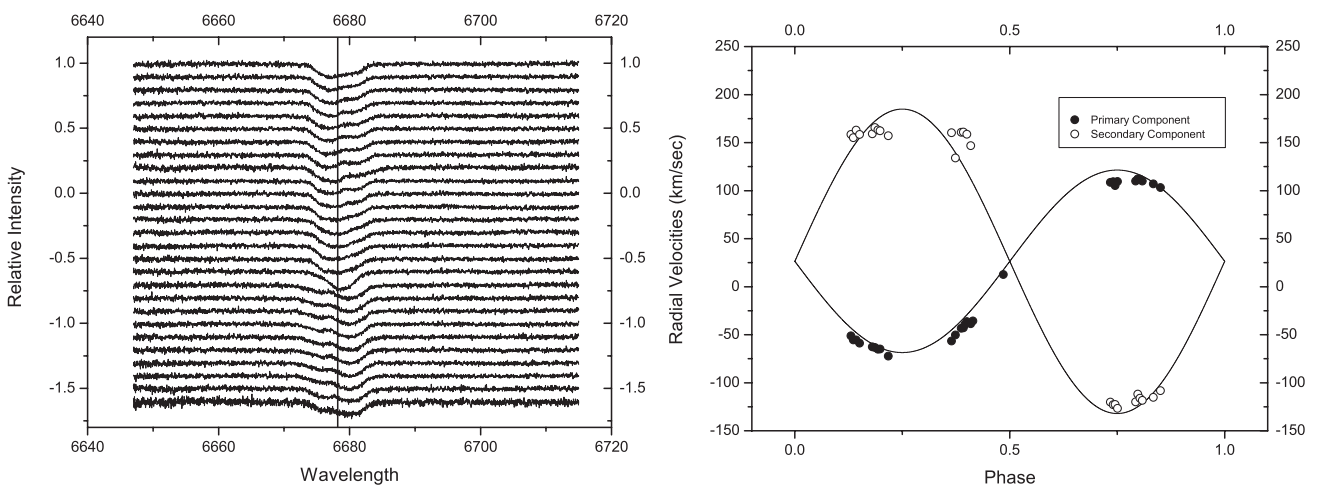

Figure 1. Left panel: The He I lines (échelle order no 85) of both components of HX Vel A were used for RVs measurements. The vertical line represents the laboratory wavelength of He I line $(6678.151 \AA)$. Right panel: Best theoretical fit to the radial velocity curves of HX Vel A. Solid line fitted to the radial velocities represents the theoretical fit for the pure Keplerian orbit.

spectral regions (containing HeI lines) where the secondary star's spectral lines could be detected. The information about the spectral regions used is given in Table 1.

The ELEMDR77 program, developed by T. Pribulla, was used to obtain orbital parameters from the radial velocity data presented here. The best fitting orbital elements are given in Table 2; the best fits to the composite spectra (left panel; showing échelle order 85, including He I line, as example) and RVs (right panel) are presented in Fig. 1.

In our forthcoming work, the physical parameters of HX Vel A will be examined, together with available light curves of the system.

\section{References}

Hearnshaw, J. B., Barnes, S. I., Kershaw, G. M., Frost, N., Graham, G., Ritchie, R., \& Nankivell, G. R. 2002, ExA, 13, 59

Skuljan, J. \& Wright, D. 2007, HRSP Hercules Reduction Software Package, vers. 3, Univ. Canterbury, New Zealand 